\title{
Avant-Garde Femtosecond Laser Writing
}

\author{
Peter G. Kazansky and Martynas Beresna \\ Optoelectronics Research Centre, University of Southampton, SO17 1BJ, United Kingdom \\ pgk@orc.soton.ac.uk \\ Yasuhiko Shimotsuma and Kazuyuki Hirao \\ Department of Material Chemistry, Graduate School of Engineering, Kyoto University, Kyoto, Japan 615-8510 \\ Yuri P. Svirko \\ Department of Physics and Mathematics, University of Joensuu, FI-80101, Finland \\ Selçuk Aktürk \\ Department of Physics, Istanbul Technical University, Maslak 34469 Istanbul, Turkey
}

\begin{abstract}
Recently discovered phenomena of quill and non-reciprocal femtosecond laser writing in glasses and crystals are reviewed. Common beliefs that laser writing does not change when reversing beam scan or propagation direction are challenged. (C) 2010 Optical Society of America

OCIS codes: (140.3390) Laser materials processing, (320.7120) Ultrafast phenomena
\end{abstract}

Ultrafast-lasers material processing has attracted considerable interest due to a wide range of applications including laser surgery, integrated optics, optical data storage, 3D micro- and nanostructuring. Three different types of material modifications can be induced with ultrafast laser irradiation in the bulk of a transparent material, silica glass in particular: an isotropic refractive index change (type 1); a form birefringence associated with self-assembled nanogratings and negative refractive index change (type 2) [1,2]; and a void (type 3). In fused silica the transition from type 1 to type 2 and finally to type 3 modification is observed with an increase of fluence.

Recently, a remarkable phenomenon in ultrafast laser processing of transparent materials has been reported manifesting itself as a change in material modification by reversing the writing direction (Fig.1) [3]. The interpretation of the phenomenon is based on anisotropic plasma trapping and heating by a tilted front of the ultrashort laser pulse. Writing direction dependence was also observed in shear stress of femtosecond laser induced lines [4].

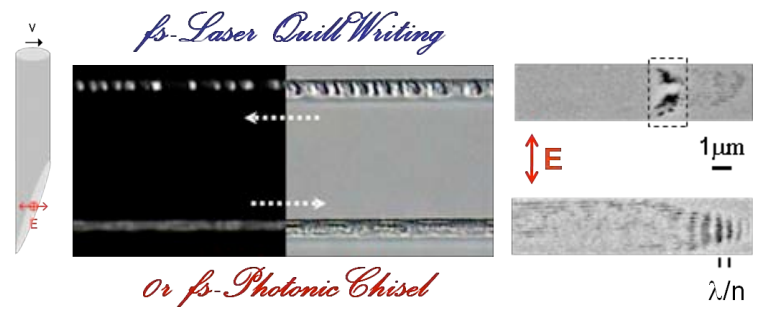

Fig. 1 (centre) Bright field images (light part) and images in crossed polarizers (dark part) of the lines written in opposite directions with amplified Yb fiber laser (left). The tilted front of the pulse along writing direction is shown. (right) SEM images of cross sections of lines written with polarization perpendicular to writing direction are also shown. The nanograting of about can be seen only in the initial part of cross sections of lines written in one of two directions. The region of collateral damage is marked with dashed line.

More recently, we have experimentally demonstrated that indeed the pulse front tilt [5] can be used as a new tool material modification control and optical manipulation, e.g. for achieving calligraphicstyle laser writing, when the appearance of a "stroke" varies in relation to its direction [6].

It has also been a common belief that in a homogeneous medium, the photosensitivity and corresponding light-induced material modifications do not change on the reversal of light propagation direction. Recently, we have demonstrated that when the direction of the femtosecond laser beam is reversed from $+Z$ to $-Z$ directions, the structures written in a lithium niobate crystal are mirror images when translating the beam along the $+Y$ and $-Y$ directions (Fig. 2) [7]. In contrast to glass, the directional dependence of writing in lithium niobate depends on the orientation of the crystal with respect to the direction of the beam movement and the light propagation direction. We demonstrate theoretically that interplay of the crystal anisotropy and light-induced heat flow gives rise to a new nonreciprocal nonlinear optical phenomenon, nonreciprocal photosensitivity. In the lithium niobate, the nonreciprocal photosensitivity manifests itself as a changing the sign of the light-induced current when the light propagation direction is reversed. Therefore, in a non-centrosymmetric medium, modification of the material can be different when light propagates in opposite directions. Non-reciprocity is 
produced by magnetic field (Faraday effect) and movement of the medium with respect to the direction of light propagation: parallel (Sagnac effect) or perpendicular (KaYaSo effect).
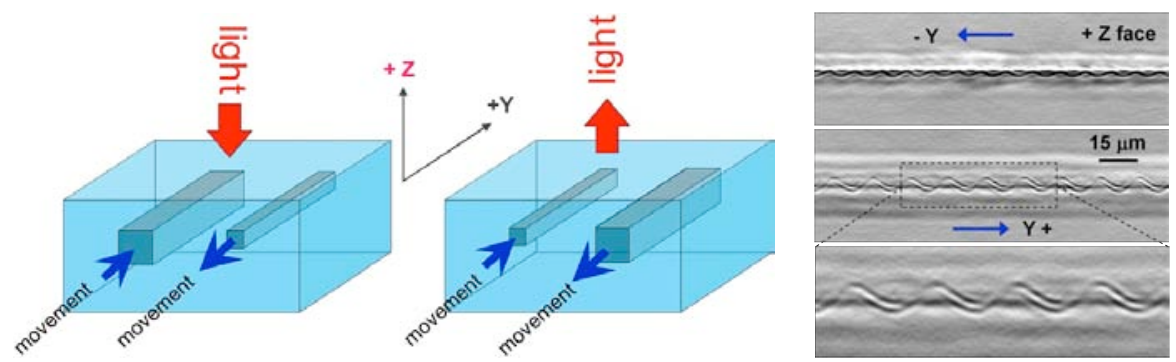

Fig. 2 The illustration of non-reciprocal laser writing - KaYaSo effect ((left and centre) and DIC images of wavy selforganized structures created when writing along the $-\mathrm{Y}$ axis and the $+\mathrm{Y}$ axis in lithium niobate (right).

More recently it has been demonstrated that refractive index and absorption induced by intense ultrashort light pulses with tilted front in an isotropic medium can be controlled with polarization plane orientation (Fig. 3) [8]. Moreover polarization dependent distribution of modifications with unusual features along the light propagation direction is observed.

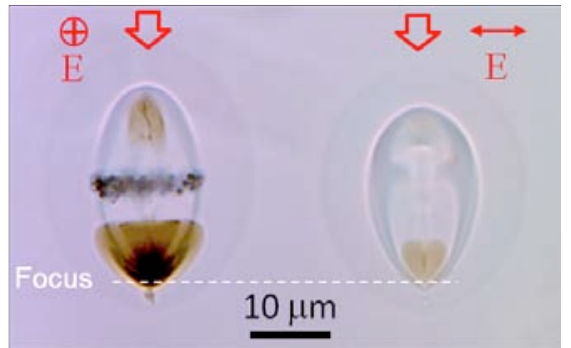

Fig. 3 Images of modified regions along beam propagation direction for writing beam polarised along the PFT (left) and in the orthogonal direction (middle). The white dashed line indicates location of geometrical focus.

We also demonstrated that self-assembled nanostructuring with femtosecond laser could be used for imprinting complex images and 4D data storage in transparent materials (Fig. 4). If sufficient retardance is achieved birefringent structure can be coloured with crossed polarizers.
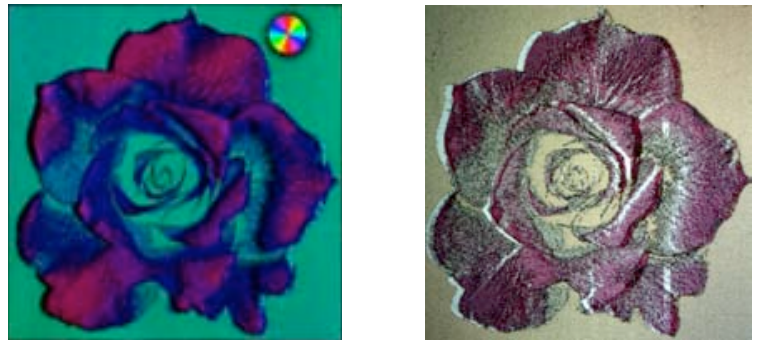

Fig. 4 Images of rose imprinted by femtosecond laser self-assembled nanostructures in silica glass. Color represents the azimuth of slow axis corresponding to the orientation of induced nanogratings (left). Optical image in crossed polarizers (right). Coloration is achieved by putting liquid crystal compensator into microscope light beam path.

We anticipate that the observed phenomena will open new opportunities in laser material processing, laser surgery, optical manipulation and data storage.

\section{References}

[1] Y. Shimotsuma, P. G. Kazansky, J. Qiu and K. Hirao, "Self-organized nanogratings in glass irradiated by ultrashort light pulses," Phys. Rev. Lett. 91, 247705 (2003).

[2] V. Bhardwaj, E. Simova, P. Rajeev, C. Hnatovsky, R. Taylor, D. Rayner and P. Corkum, "Optically produced arrays of planar nanostructures inside fused silica," Phys. Rev. Lett. 96, 057404-1 (2006).

[3] P. G. Kazansky, W. Yang, E. Bricchi, J. Bovatsek, A. Arai, Y. Shimotsuma, K. Miura and K. Hirao,"Quill" writing with ultrashort light pulses in transparent materials," Appl. Phys. Lett. 90, 151120 (2007).

[4] B. Poumellec, L. Sudrie, M. Franco, B. Prade, and A. Mysyrowicz, "Femtosecond laser irradiation stress induced in pure silica," Opt. Express 11, 1070-1079 (2003)

[5] S. Akturk, M. Kimmel, P. O'Shea, and R. Trebino, "Measuring spatial chirp in ultrashort pulses using single-shot FrequencyResolved Optical Gating," Opt. Express 11, 68-78 (2003).

[6] W. Yang, P. G. Kazansky, Y. Shimotsuma, M. Sakakura, K. Miura and K. Hirao, "Ultrashort-pulse laser calligraphy,” Appl. Phys. Lett. 93, 171109 (2008).

[7] W. Yang, P. G. Kazansky and Yu. P. Svirko, "Non-reciprocal ultrafast laser writing," Nature Photonics, 2, 99-105 (2008).

[8] P. G. Kazansky, Y. Shimotsuma, J. Qiu, M. Sakakura, M. Beresna, Yu. P. Svirko, S. Akturk, K. Miura and K. Hirao, "Ultrafast light blade: Anisotropic sensitivity of isotropic medium to femtosecond laser radiation", submitted to PRL(2010). 